\title{
Bending techniques for flat materials using cut patterns: a review
}

\author{
Barbara Ruschel Lorenzoni * and Fabio Pinto da Silva \\ Design and Materials Selection Lab - LDSM, Federal University of Rio Grande do Sul - UFRGS, Av. Osvaldo Aranha, 99 - sala \\ 604, 90035-190, Porto Alegre, RS, Brazil.
}

Global Journal of Engineering and Technology Advances, 2021, 07(02), 091-102

Publication history: Received on 12 April 2021; revised on 20 May 2021; accepted on 22 May 2021

Article DOI: https://doi.org/10.30574/gjeta.2021.7.2.0070

\begin{abstract}
The techniques that permit the materialization of organic and curved geometries include those based on curving flat materials using cut patterns, which have been explored with the improvement of digital manufacturing tools. This study has analyzed kerfing, lamina emergent mechanisms, auxetic linkages and kirigami, seeking greater knowledge and differentiation between these techniques. The mechanical principles, objectives, materials and possible applications, types of cutting patterns, among other aspects, were investigated. It was noted that kerfing and lamina emergent mechanisms presented similar aspects with regards to aiming at folding or bending the material, the use of materials with considerable thickness, and the dependence of the torsion of segments for bending the set. Meanwhile, auxetic linkages and kirigami were also used for stretching the material and depended on buckling or bending of segments, being more suitable to thin or flexible materials. For kerfing, the focus was on architectural scale applications, while kirigami was used in small scale applications (such as electronics). No specific applications were identified for LEMs and auxetic linkages. The information collected and the understanding of each system sought to contribute to a greater knowledge and adoption of these techniques by designers.
\end{abstract}

Keywords: Cut pattern; Bending flat materials; Kerfing; Lamina emergent mechanisms; Auxetic linkages; Kirigami

\section{Introduction}

Advances in digital tools for design and architecture have resulted in great design freedom, encouraging designers to idealize complex and organic forms. However, this phenomenon was not accompanied by the development of platforms that combine this freedom with constructive aspects [1-8].

In the physical world, unlike the digital one, the material limitations are inseparable from their forms, and their behavior is a decisive factor in defining the geometries [8,9]. In addition, industrial materials have limitations in size, shape and stiffness due to requirements for mass production methods [8].

Structure, properties and processing influence each other, reflecting on the possibilities of material application. The structure is the three-dimensional arrangement of the atoms that compose it, while its properties represent qualities that are provided both by the structure and by its chemical composition, distinguishing it from other materials. Some of the typical mechanical properties of a material include stiffness and yield stress - in tension, compression and shear rupture stress, etc., and the technological properties, workability, weldability and possibility of recycling, which influence the manufacturing aspects. Materials processing is defined as the series of steps used in the manufacture of raw-materials into finished goods to obtain a certain shape and modify the material structure and, consequently, its properties [10].

\footnotetext{
* Corresponding author: Barbara Ruschel Lorenzoni; phone: +55 51 999841090; e-mail: brlorenzoni@gmail.com Design and Materials Selection Lab - LDSM, Federal University of Rio Grande do Sul - UFRGS, Av. Osvaldo Aranha, 99 - sala 604, 90035-190, Porto Alegre, RS, Brazil.
} 
Therefore, the steps used to modify these materials by altering their macrostructure are investigated in an attempt to confer apparent properties distinct from the original ones, and thus, manipulate their behavior [1, 11, 12]. The geometric manipulation of materials can be an example and gives rise to metamaterials, which are created from geometric concepts [13].

Among the interventions based on geometric parameters, there are those that operate by cutting or subtracting materials and that allow curving or transforming flat substrates into three-dimensional geometries. These studies have shown potential due to the advancement of digital manufacturing equipment, given the possibility of performing precise and complex cut patterns at high velocity and precision, allowing an unprecedented capacity to bend some materials. According to Cho et al. [13], the majority of materials does not have much elastic elongation and are limited to geometries to which they can deform. However, it is possible to allow greater stresses and changes in macroscopic shape in any material, using cut patterns.

This way of transforming two-dimensional materials into three-dimensional geometries has inspired studies in different areas and scales, which is partly due to the possibility of using flat materials processing tools (materials that are commonly accessible, easy to store and transport), creating new possibilities for uses and functions in many different areas. However, it is necessary to have great knowledge of these techniques so that they can become programmable, predictable and accessible to designers and architects.

Therefore, this study aimed at investigating the techniques for bending flat materials using cutting patterns in order to differentiate them, facilitating their understanding and use. The techniques will be analyzed for their principles of mechanical behavior, types of applicable materials, scales and application possibilities and other specific and potential characteristics.

\section{Methodology}

In order to understand and differentiate the techniques for bending flat materials using cutting patterns, a review was made on the topic, based on the methodology proposed by Kitchenham and Charters [14]. After a primary search for data capture and filter definition (carried out in Science Direct and Scopus), a secondary search was performed between March 20 and May 31, 2019 in the Science Direct, Scopus and Google Scholar databases. The search interval was defined from 2005 to 2019. The strings consisted of synonyms: relief cut, kerfing cutting, kerf cut, kerfing pattern and kerf for the technique; flat, sheet and panel for the material; and curving, bending, folding, flexible and elasticity for the expected result. No field or search source was filtered, except patents and citations.

In addition to using search filters, periods and databases, the studies were also searched by the title, being excluded those cases not related to the topic. For the remaining studies, the keywords and summary were read. Studies without original translation into Portuguese or English and duplicate ones were excluded. The fourth and last searches started from the full reading of the papers, from which it was considered pertinent the exclusion of those that mentioned the technique without investigating it, those referring to materials stretching, those fully contained in others, and finally, the papers in which the adopted technique was not crucial for bending materials.

The review allowed the recognition of some techniques used to bend flat materials, namely: kirigami, auxetic linkages, lamina emergent mechanisms (LEMs) and kerfing. The grouping of techniques was done by the author of this study, according to the denomination given by the author of each article or, and when not provided, by similarity to other studies. In the items that follow, the techniques were conceptualized according to the material found. At the end, a synthesis was made for comparison and differentiation of the techniques.

\section{Techniques for bending flat materials using cut patterns}

\subsection{Kirigami}

Kirigami is a japanese art technique that results in complex three-dimensional shapes made of cut paper [15]. This technique seeks to respond to challenges, such as the involvement of a three-dimensional structure with a flat material, without deformations or increased bearing stress and without changing its intrinsic properties. Due to the stiffness of most device components, it is necessary to insert cuts into them to make acute folds possible without shearing and stretching the material. The cuts consist of simple lines or any geometry to form arrays of cuts with different purposes [16]. 
To obtain great stretching of material, structures with programmable stresses have been used from manipulating the geometry of the kirigami cuts and consequently predicting the mechanical deformations of the materials and controlling their properties $[17,18]$. These properties can be related to the structure of the material (such as macrogeometry and the pore shape), mechanical aspects (such as maximum stress), and even properties associated with some structures (electrical, acoustic, photonic, etc.) [13]. In this way, the performance of materials is improved, especially with regard to flexibility [19]. Thus, kirigami allows the combination of properties that usually contradict each other (such as high conductivity and material stretching).

The studies on kirigami structures search for innovative technical solutions in stretchable and wearable electronic devices [17, 19, 20,21], biomedical tools [22], energy capture and storage devices [20, 23], soft-robotics [24], among others. It is important to highlight the potential detected in kirigami for use in flexible electronics. Materials conventionally used for electronics are not able to adapt their shape in response to external stimuli. As an alternative, kirigami makes it possible to maintain the conductivity of the material in combination with increasing its stretching capacity $[17,18,21]$.

Kirigami allows an apparent elasticity to the material, facilitating the obtainment of developable surfaces. In addition, the possibility of extension in the plane allows the metric distortions necessary to conform surfaces of apparent intrinsic curvature in a global sense (since the materials remain intrinsically flat) due to deformations in small faces connected with each other [25].

Shyu et al. [18] suggest that the kirigami rules apply to different scales and materials in which high deformations are desired. Hwang and Bartlett [26] emphasized that these geometric deformations that interfere with the flexibility of the material and structure resulting from the technique are determined by the pattern and orientation of the cuts. In addition to the cut design, a crucial parameter in kirigami is the one that translates the relationship between in-plane stiffness and out-of-plane bending stiffness, with materials that are more easily bent than sheared or stretched those that obtain higher numbers [15], and therefore, material thickness is also extremely relevant [15, 25]. In general, the art is applied to thin and unstretchable flat materials $[19,25]$. For some authors, the kirigami technique achieves better results with flexible substrates $[13,27,28,29,30]$.

Three variations of the kirigami technique were observed in the literature: lattice kirigami, kirigami by fractal cuts and by parallel cuts. Yang, Choi and Kamien [16] described lattice kirigami as the one realized by embedding an array of cuts into a thin sheet in which the edges are brought together by folding it, obtaining staggered surfaces. As it involves folding, this variant has not been further investigated.

Kirigami of fractal cuts is based on the division of the material by cuts in rotational polygons of geometry dictated by the cut pattern. During the application of loads on the material, the polygons function in an approximately rigid way, undergoing mostly rotation, while the connections between them function as hinges with high rotational freedom by bending $[13,16,25]$, observed in Figure 1 . The final geometry of the material is determined by the angles between polygons. It is possible to manipulate the material by applying different motifs and hierarchical levels of cut [13], forming arrays of cuts as simulated in Figure 2. Fractal cuts allow the approximation of surfaces with intrinsic curvature by biaxial stretching and compression of the material [25]. This curvature occurs in the empty areas, since the polygons remain flat [27]. If a non-developable surface is sought, a non-uniform extension of the material will occur, resulting in a distinct opening of the cuts [13]. 
a

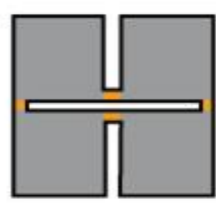

c

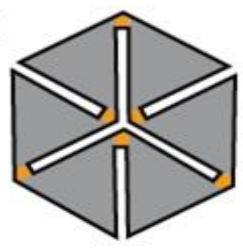

b

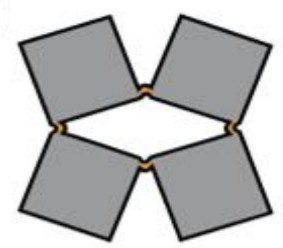

polygonal units

joints d

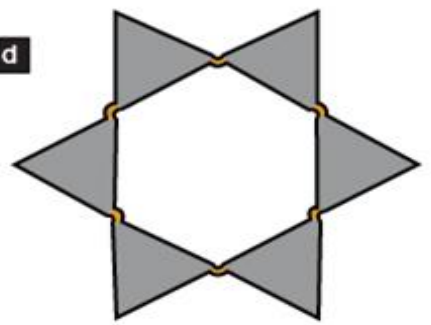

Figure 1 Schematic illustrations of fractal kirigami patterns of square polygons on the original shape (a) and on the stretched shape (b) and of triangle polygons on the original shape (c) and on the stretched shape (d).

a

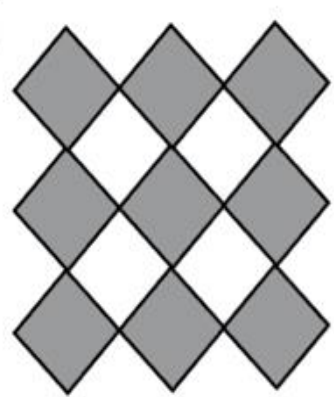

b

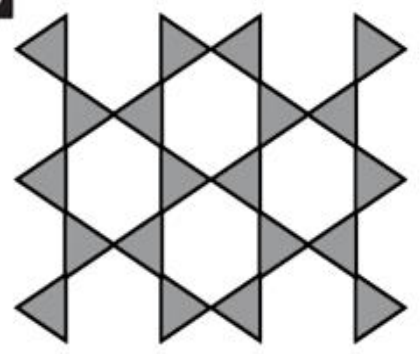

Figure 2 Schematic illustrations of arrays of fractal kirigami of square (a) and triangle (b) polygons.

In fractal kirigami, some elements can be fixed on rotational units without losing their functional properties. The technique is applicable to several scales and materials, as long as a joint behavior is possible (such as occurs in elastomers and metals), keeping the stresses in the joints lower than the material limit. Therefore, it is necessary to pay attention to the selection of materials in relation to bending stiffness and fracture and yield strength [13].

The parallel-cut kirigami (Figure 3) allows deformation by uniaxially applied tensile stresses that accommodate high macroscopic deformation even on rigid substrates [30,31]. Chen et al. [17], for example, used this technique and obtained elasticity larger than $277 \%$ in kirigami metallic glasses. According to Xu, Shyu and Kotov [31], this occurs due to the difference in bending stiffness and stiffness in the material plane, characteristic of thin flat materials, favoring out-of-plane deformation to minimize stress energy. [25] emphasized the importance of thickness in this system, since it depends on the material buckling. Note also the possibility of maintaining important properties of the materials used, even with high elongation rates [18]. 
a

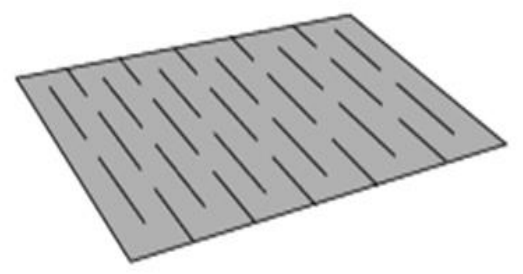

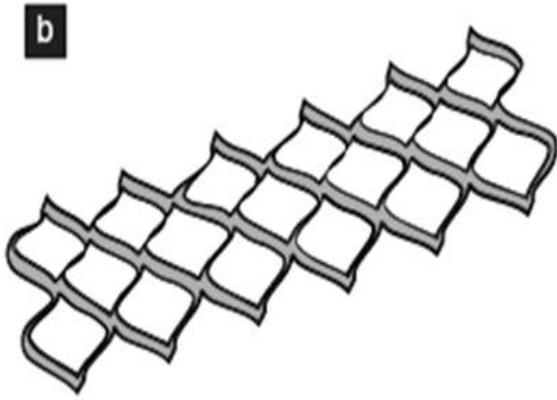

Figure 3 Schematic illustrations of parallel-cut kirigami in original format (a) and when stretched (b).

There are also studies of kirigami of parallel cuts to obtain pop-up structures [32]. These occur from the application of load perpendicular to the material plane, with buckling segments resulting in three-dimensional structures.

\subsection{Auxetic linkages}

Auxetic materials are those that have negative Poisson's ratio. These materials present an increase in width when stretched and a decrease when compressed. This unexpected behavior is attributed to two factors: the geometry of the material and its internal structure. One of the potentials of these materials is the optimization of some characteristics by altering their spatial distribution, which adds to their intrinsic properties. Some examples include improvement of mechanical properties (such as stiffness), insertion of anisotropy in industrial materials and acquisition of synclastic curvature. In addition, there are some materials and structures that are developable due to an auxetic property [33].

For Konaković et al. [34], auxetic linkages are auxetic materials manufactured by introducing a specific pattern of cuts into approximately inextensible material such as sheet metal, or polymers, which, when stretched, rotate the polygons formed by the cuts in relation to its neighbors, so that the material expands evenly. The authors [34] associated this characteristic to the ability of these materials to obtain surfaces with non-zero Gaussian curvature.

Auxetic behavior is achieved by specific topologies, such as reentrant geometry units, rigid or semi-rigid rotational units, spiral systems, circular shapes forming voids, among others [30, 35, 36, 37, 38], some illustrated in Figure 4. However, according to the study by Louth et al. [39], there are some limitations in obtaining a developable surface by certain auxetic cut patterns.
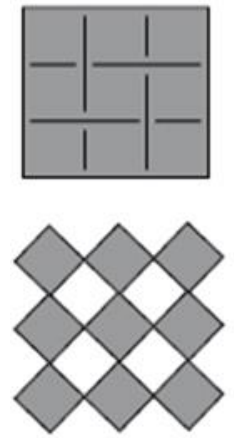
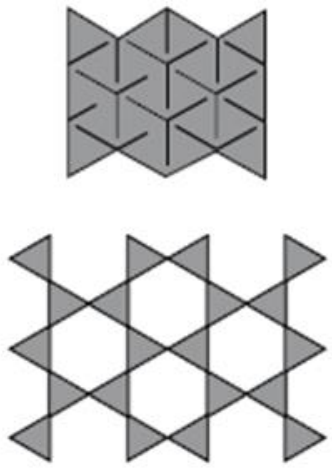
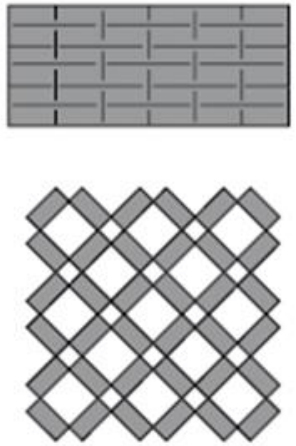
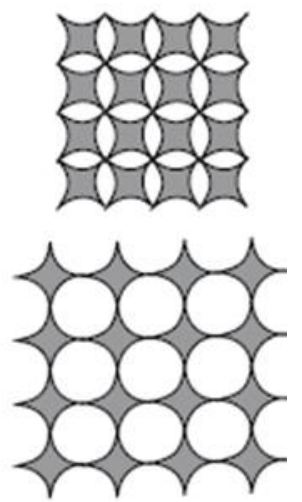

Figure 4 Geometries with auxetic behaviour in the original and stretched shape.

Similar to fractal cut kirigami, when the material with cuts forming polygons is expanded, the polygons remain practically rigid, concentrating the deformation at the joints, which do not resist external forces and allow the mesh to be opened isotropically, and as a result, the surface scale change is absorbed without great shearing effort. There is, however, a stress limit that the joints can tolerate without fracturing. It varies for each material, thickness and depth of the cuts, so that there is a maximum stretching depending on both the substrate and its geometry [34]. 


\subsection{Lamina emergent mechanisms (LEMs)}

Some studies have investigated lamina emergent mechanisms (LEMs) (Figure 5), which are mechanisms fabricated from planar materials (lamina) that emerge out of the fabrication plane $[40,41]$, functioning as joints. According to Jacobsen et al. [41], through simple topology obtained from common manufacturing processes of flat materials, these mechanisms are capable of performing complex mechanical motions, and according to Nelson et al. [42], its primary motion is similar to a fold.
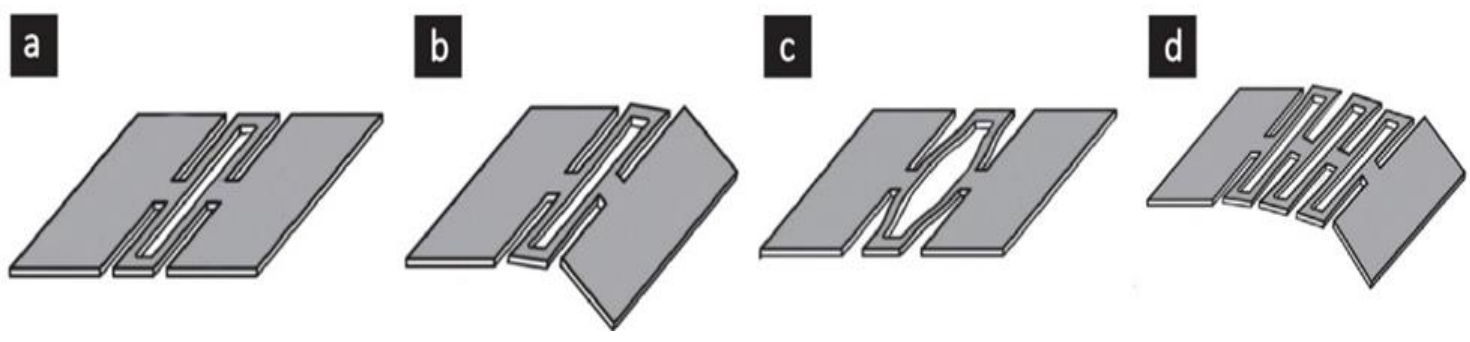

Figure 5 Illustration of (a) simple LEM; (b) LEM in bending movement; (c) LEM in tension or stretching; (d) Array of LEMs.

LEMs motion is partly due to the deflection of flexible members, which means that they can be included in the group of compliant mechanisms [42, 43, 44, 45]. There are also similarities between origami systems and LEMs [41, 42, 44, 46, $47,48]$, which can be considered a surrogate fold. These surrogate folds alter the boundary conditions of the material at the joint sites, changing its apparent properties and reducing its stiffness, so that movements equivalent to the fold can occur without fracture $[47,48]$.

LEM-type mechanisms can be based on different means, which may interfere with the apparent flexibility of the material: geometric change (width, thickness or length of segments or cross sections), changes in material properties (such as materials with heterogeneous properties in space, changing boundary conditions by changing the fixation or the loading conditions) or by creating hybrid components [41]. In the case of LEMs based on geometric changes, the complex motion observed is due to a combination of fundamental motions made by their segments. As for the load conditions, they may incur bending, axial tension, torsion or a combination of these [40]. Some motions resulting from this system may be desirable or not, and the most common type is the one that curves the material by the torsion of its segments [47].

Some advantages of LEMs over other types of joints are: manufacture from flat materials; reduction of production, storage and transportation costs; reduction of parts, which simplifies manufacturing, eliminates the friction wear and tear, reduces the weight of the structure, facilitates recycling and precision, eliminates assembly and has a more integrated design $[40,41,42,46]$. Possible applications for LEMs are: limited space mechanisms (such as electronics and medical equipment), equipment that require compact transportation (such as aerospace, automobile, naval, furniture items and emergency shelters), and applications that have limited manufacturing (such as microelectronics) $[41,42]$.

According to Nelson et al. [42], LEMs can be applied to various materials using additive and subtractive manufacturing technologies. However, those with a better relation between the yield limit and Young's modulus have a greater flexibility response [40].

There are several types of LEMs, some of which allow a simple bending movement but facilitate the high concentration of tension in the bending elements, reducing the bending capacity of the assembly. Conversely, those joints that have their segments parallel to the axis of rotation, based on their torsion, have the advantage of allowing high deflections of the joint, maintaining constant stiffness, and reducing joint stress, however, they are more susceptible to unwanted movements in compression and tension, which generate bending of these elements. This susceptibility increases with the increase in the length of the torsion segments, despite the fact that a greater angle of curvature of the set is also obtained. There is also a tendency that the smaller the width of the joints, the more precise the obtained fold, however, the resistance to compression and tension is reduced, so that joints designed to resist it generally have a greater width and, as a consequence, an unstable center of rotation. Another movement that can be expected in a joint is lateral bending, however, it is difficult to obtain it without also allowing joint twisting [47]. 
The distribution of LEMs in a network results in a lamina emergent array [42], which allows a much greater movement than each individual element [47]. The arrays consist of panels in which the joints (geometries) are distributed along the axis of rotation (in parallel) or in the direction of moving away from the axis (in series), or both, and are adaptable to different scales $[42,44,47]$. When the LEMs are arranged in an array, the deflection angles of the set are calculated from the deflection angle of each joint unit [42].

LEM arrays can also be considered modified materials, since they are manufactured from a single material piece and alter certain apparent properties of a material (in general the bending stiffness outside the plane), resulting in high elastic deformation. The combination of similar or different LEMs generates different arrays that can be applied to obtain developable surfaces, including in thick materials [44], and also approximate desired three-dimensional surfaces [42].

\subsection{Kerfing}

Kerfing is a technique for bending rigid materials that is based on cutting, or subtracting, some areas of flat substrates in order to alleviate the stress caused by the bending effort in the material (of compression on the concave face and tension on the convex face of the curve) $[3,4,8,49,50,51,52,53]$, as shown in Figure 6 . This technique can be used to curve those materials and also to conform to three-dimensional structures from a continuous panel. The technique is also found in the literature under the terms relief cutting, living hinge, lattice hinge, kef-bending and dukta [4, 8, 49, 54].
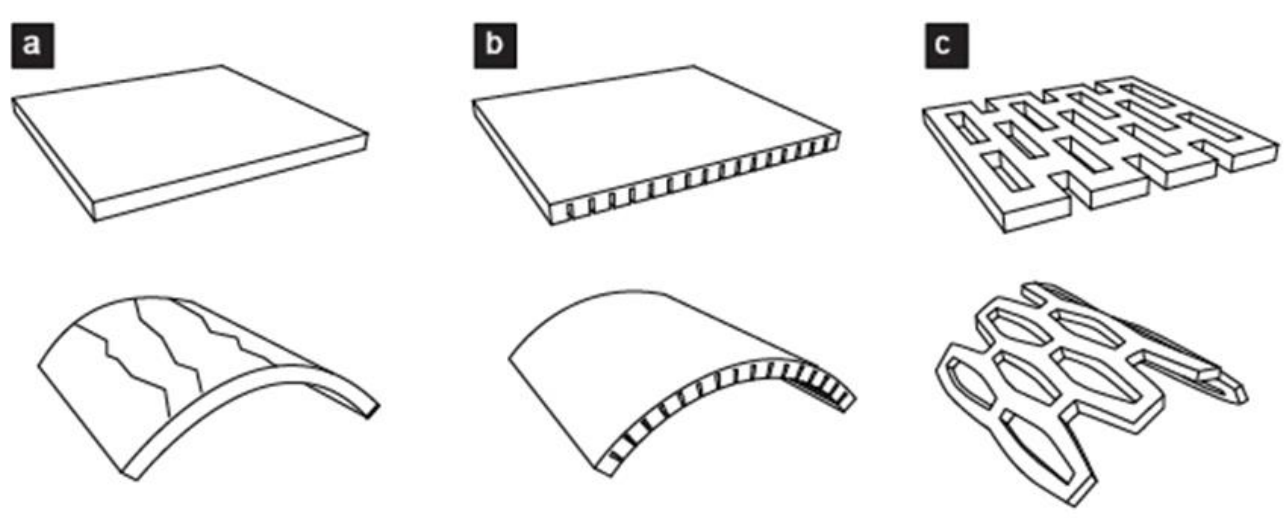

Figure 6 Representations of what happens when trying to bend: (a) a rigid panel (b) a rigid panel with kerfing on one side of the panel (c) a rigid panel with kerfing through the panel thickness.

As an example of the combination of properties of both the material (particularly its Young's modulus and thickness, which affect other properties) and its shape [54], kerfing is a subtractive manufacturing method that alters the strength of the material and increases its apparent flexibility [49]. In many cases, such as Borhani and Kalantar [2], the technique aims to meet situations in which contradictory attributes are necessary, such as the simultaneous need for high elastic deformation and rigidity to support load. One of its advantages is that, by combining it into a single component, the number of parts, assembly time and maintenance costs are reduced [55], in addition to facilitating transport and storage in the flat format $[49,50]$.

Kerfing is usually applied to wood, MDF, cardboard, polymers and metals [54], aiming largely at architectural purposes, such as acoustic panels [56], systems for facades and roofs [55], furniture [49, 57, 58], structural formworks [4], decorative or structural panels [1, 50,52], integrated floor, wall and roof structures [11], among others. One of the disadvantages of this technique is that, due to the reduced stiffness and strength of the resultant material, it may not be suitable for situations that require significant structural performance [11, 49, 59], being more appropriate to applications that explore their aesthetic potential, as in Capone and Lanzara [49], Holterman [56], Jensen, Blindheim and Steinert [60], Mitov et al. [52] and Wei and Singh [61].

The main mechanical effort responsible for the apparent flexibility of the kerfing materials is the torsion of the segments parallel to the bending axis, when the set is bent $[3,55,56]$. A minor bending movement also occurs, but in the segments perpendicular to the rotation axis. The total curvature of the surface represents the sum of the small angles that each segment is capable of rotating $[4,55,56]$. According to Capone and Lanzara [49], the limiting factor of the deformation 
is the maximum torsional stress of the segments, which depends on the material, thickness, shape and size of the cuts. The mechanical effort related to each segment of the kerfing material can be observed in Figure 7.
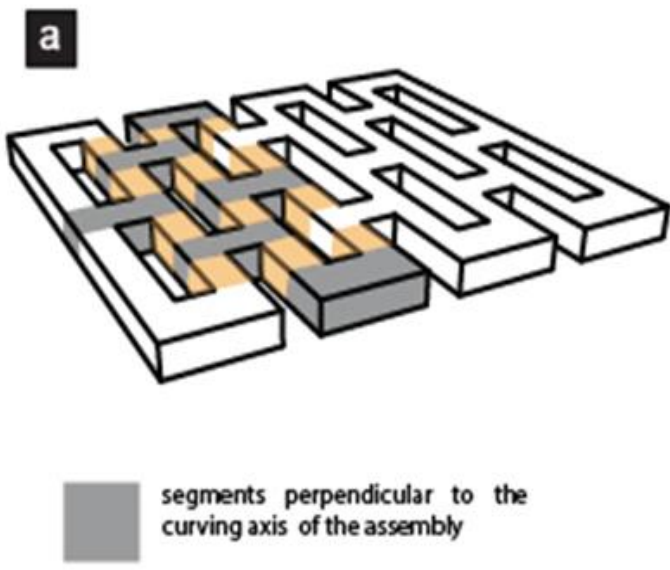

segments perpendicular to the curving axis of the assembly

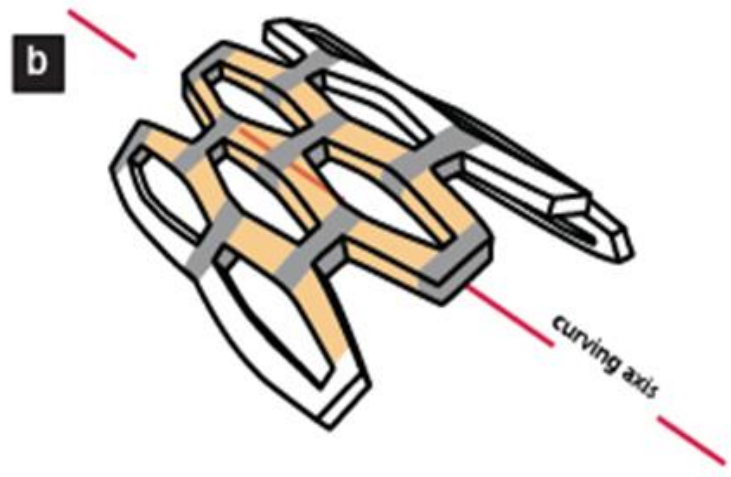

segments parallel to the curving axis of the assembly

Figure 7 Schematic illustrations of the panel with kerfing in flat (a) and curved (b) positions.

The geometric properties of the cut pattern affect the bending outcomes [54]. There are different types of kerfing depending on the face of application (side of the material on which the cuts are applied) and the cut pattern (geometric design of the incisions). Kerfing applied on one side of the material (original from traditional wood-working) leaves part of its thickness, maintains a continuous face and allows only developable surfaces [4, 50, 52, 55]. Kerfing applied on both sides of the material using perpendicular cuts results in a structure that can bend and twist and be used to configure a double curvature surface $[1,49]$. In contrast, the type of kerfing that passes through the material thickness is able to accommodate tension and compression in the direction perpendicular to the applied force, depending on the cut pattern [55]. There are several kerfing patterns that pass through the material thickness, depending on the geometric shape of the cuts and the type of curvature allowed by them $[49,57,59,60]$. In this case it is possible to observe some predominant cut patterns: cuts made up of parallel linear incisions in series, with one or more segments perpendicular to the material curving axis arranged in an interspersed manner $[11,49,54,55,56,58,62]$ and interlaced spiral cuts that allow for bending in more than one direction $[2,3,4,8,53,56,59,62]$.

\section{Discussion}

The review of the studies showed that the different techniques overlap or are related. There is, however, a difference between the main objectives of each technique in relation to the desired movement or geometry: kerfing is used to bend the material; kirigami enables a material to stretch far beyond what its normal tensile properties would allow; auxetic linkages are materials that undergo lateral expansion when stretched and become thinner when compressed; and LEMs are mechanisms fabricated from planar materials (lamina) that emerge out of the fabrication plane and allow the material to fold and possibly curve (for LEM arrays). In this sense, it is possible to identify similarities between kerfing and LEMs and between kirigami and auxetic linkages, in both the objectives and the mechanical efforts that each technique causes in the material.

Despite the differences observed, Greenberg [46] showed similarities between LEMs and kirigami, and the kinetic models of the latter, especially of the pop-up type, which are often LEMs. Zarrinmehr et al. [53] found that techniques based on cutting the material to obtain flexible panels, such as kerfing, depend on the auxetic behavior resulting from the cuts, which occurs through rotation of the polygons formed between them. In turn, Callens and Zadpoor [25] verified a similarity between kirigami and auxetic metamaterials. Celli et al. [63] investigated the auxetic effect on some cases to obtain three-dimensional geometries.

There were differences in predicted functions regarding the materials, which in studies on kirigami were predominantly focused on flexible electronics, and kerfing architectural applications. In kirigami applications, this was attributed to the maintenance of material properties in parallel cuts; in fractal cuts, to the possibility of positioning electronic elements over rigid polygons. In addition, it was noted that for kirigami, one of the objectives was to assess how the material 
behaved when subjected to load application and removal efforts, which did not occur in most kerfing cases, used mostly for static applications. The studies on auxetic linkages and LEMs were investigative and did not focus on specific applications.

Differences between the materials that are more appropriate to each technique were also observed and were defined according to the mechanical efforts required. The kirigami of parallel cuts is more suitable for thin or flexible materials as it is based on the buckling of the segments for the elongation of material $[15,19]$. In a similar way, because it depends on the bending of joints between rotational polygons, fractal kirigami and auxetic linkages require a material that allows this behavior, such as flexible substrates $[13,27,28,29,30]$. LEMs are not geared to a specific material, and different types of LEMs (which are based on different demands) can be applied to different materials. Conversely, kerfing can be applied to rigid and thick substrates that do not tolerate bending or buckling easily because it depends mainly on the torsion of the material segments to bend the set.

Regarding the cut pattern, on kerfing, LEMs and kirigami studies there is a preference for straight lines with interruptions (forming segments perpendicular to the material bending axis) crossing the thickness of the material [11, $49,55,56,62]$. With regards to kerfing and LEMs, however, a portion of the material is commonly removed around the cut line as the techniques involve thick materials, thus preventing it from intersecting itself when bent. For parallel cut kirigami, however, cut patterns in which the material close to the line is removed are uncommon, since the intention is to stretch the material with minimum thickness. In auxetic linkages studies, cut patterns forming internal squares (also common in fractal kirigami) are more frequent. It was possible to observe that while the studies that included linear cuts focused mainly on the uniaxial extension behavior or simple curvature, those that included fractal cuts or spirals focused on biaxial expansion, or bending in more than one direction.

\section{Conclusion}

Flat materials bending techniques obtained from cut patterns analyzed by this review presented the same basic principle of operation, which consisted of altering the apparent properties of these materials by geometric interventions. In some respects, the techniques were similar and in certain situations they may even coincide, however, it was observed that there were fundamental differences as far as the objectives, the mechanical efforts involved and possible applications for each technique were concerned.

With the possibility of using digital manufacturing tools, the techniques have the advantage of bringing the project and execution closer together, thus allowing their adaptation to each situation. A better understanding of the differences between them, which was the main objective of this study, may help to choose the most appropriate for each project. In addition, it is necessary to know about the materials and mechanical design demands, and also the development of prototypes to analyze the techniques variations, as well as the cut patterns and parameters.

The referred techniques show potential in providing innovative material options for designers. There are studies analyzing the influence of cut patterns and geometric parameters on the flexibility and geometric results, which will surely improve knowledge on the subject. The understanding of intrinsic properties and characteristics of the materials, which are already known, can be inserted into digital systems for analysis and simulation of projects. Currently, experimental researches on the subject are being conducted, however, greater knowledge of the techniques is necessary to be properly integrated into digital design and manufacturing systems for a broader use.

\section{Compliance with ethical standards}

\section{Acknowledgments}

We gratefully acknowledge the financial support provided by the Coordination for the Improvement of Higher Education Personnel (CAPES, Brazil) and the National Council for Scientific and Technological Development (CNPq, Brazil). We also thank the Design and Materials Selection Lab (LDSM/UFRGS) for its contribution to this research.

\section{Disclosure of conflict of interest}

The authors have no conflicts of interest to declare. 


\section{References}

[1] Bianconi F, Filippucci M. WOOD, CAD AND AI: Digital Modelling as Place of Convergence of Natural and Artificial Intelligent to Design Timber Architecture. In: Bianconi F and Filippucci M, eds. Digital Wood Design. Lecture Notes in Civil Engineering 24. Springer, Cham; 2019. 19-23.

[2] Borhani A, Kalantar N. Transformative Formworks: Towards Mass Customization of Double-Curved Surfaces. In: Shape Modeling International. Berkeley, USA; 2017.

[3] Chen R, Jiang M, Kalantar N, Moreno M, Muliana A. Creating Flexible Structures out of MDF Plates. In: Proceedings of the 18th US-Japan Conference on the composite materials ASTM commited D30 meeting. 2018.

[4] Kalantar N, Borhani A. INFORMING DEFORMABLE FORMWORKS: Parameterizing Deformation Behavior of a Non-Stretchable Membrane via Kerfing. In: Proceedings of the 23rd International Conference of the Association for Computer-Aided Architectural Design Research in Asia, vol 2. Beijing, China; 2018: 339-348.

[5] Lawson B. CAD and creativity: does the computer really help? Leonardo (Oxf). 2002; 35(3): 327-331.

[6] Menges A. Computational Material Cultures. In: Rycke KD et al., ed. Humanizing Digital Reality. Singapore: Springer; 2018. 5-11.

[7] Sass L, Oxman R. Materializing design: the implications of rapid prototyping in digital design. Des Stud. 2006; 27: 325-355.

[8] Zarrinmehr S, Ettehad M, Kalantar N, Borhani A, Sueda S, Akleman E. Interlocked archimedean spirals for conversion of planar rigid panels into locally flexible panels with stiffness control. Computers \& Graphics. 2017; 66: 93-102.

[9] Fleischmann M, Knippers J, Lienhard J, Menges A, Schleicher S. Material behaviour: embedding physical properties in computational design processes. Architectural Design. 2012; 82(2): 44-51.

[10] Walter Y. O conteúdo da forma: subsídios para seleção de materiais e design [Master Thesis]. Bauru, SP, Brazil: School of Architecture, Arts and Comunication, São Paulo State University; 2006.

[11] Greenberg E, Körner A. Subtractive Manufacturing for Variable-Stiffness Plywood Composite Structures. In: Proceedings SDM 2014 International Conference on Sustainable Design and Manufacturing. Cardiff: Cardiff University; 2014: 50-66.

[12] Oxman N. Programming matter. Architectural Design. 2012; 82(2): 88-95.

[13] Cho Y, Shin J-H, Costa A, Lim TA, Kunin V, Lid J, Lee SY, Yang S, Han HN, Choi I-S, Srolovitz DJ. Engineering the shape and structure of materials by fractal cut. Proc Natl Acad Sci U S A. 2014; 111 (49): 17390-17395.

[14] Kitchenham B, Charters S. Guidelines for performing Systematic Literature Reviews in Software Engineering. Software Engineering Group, School of Computer Science and Mathematics, Keele University and Department of Computer Science, University of Durham, United Kingdom; 2007. 57 p. EBSE Technical Report. EBSE-2007-01.

[15] Blees MK, Barnard AW, Rose PA, Roberts SP, Mcgill KL, Huang PY, Ruyack AR, Kevek JW, Kobrin B, Muller DA, Mceuen PL. Graphene kirigami. Nature. 2015; 524: 204-212.

[16] Yang S, Choi I-S, Kamien RD. Design of super-conformable, foldable materials via fractal cuts and lattice kirigami. Material Research Society Bulletin. 2016; 41: 130-138.

[17] Chen SH, Chan KC, Han DX, Zhao L, Wu FF. Programmable super elastic kirigami metallic glasses. Mater Des. 2019; 169.

[18] Shyu TC, Damasceno PF, Dodd PM, Lamourex A, Xu L, Shlian M, Shtein M, Glotzer SC, Kotov, NA. A kirigami approach to engineering elasticity in nanocomposites through patterned defects. Nat Mater. 2015; 14: 785-790.

[19] Zheng W, Huang W, Gao F, Yang H, Dai M, Liu G, Yang B, Zhang J, Fu YQ, Chen X, Qiu Y, Jia D, Zhou Y, Hu P. KirigamiInspired Highly Stretchable Nanoscale Devices Using Multidimensional Deformation of Monolayer MoS2. Chem Mater. 2018; 30: 6063-6070.

[20] Guo H, Yeh M-H, Lai Y-C, Zi Y, Wu C, Wen Z, Hu C, Wang ZL. All-in-One Shape-Adaptive Self-Charging Power Package for Wearable Electronics. ACS Nano. 2016; 10: 10580-10588.

[21] Ma R, Wu C, Wang ZL, Tsukruk VV. Pop-Up Conducting Large-Area Biographene Kirigami. ACS Nano. 2018; 12: 9714-9720. 
[22] Zhao R, Lin S, Yuk H, Zhao X. Kirigami enhances film adhesion. Soft Matter. 2018; 14: 2515-2525.

[23] Lamoureux A, Lee K, Shlian M, Forrest SR, Shtein M. Dynamic kirigami structures for integrated solar tracking. Nat Commun. 2015; 6: 1-6.

[24] Mcevoy MA, Correll N. Shape-Changing Materials Using Variable Stiffness and Distributed Control. Soft Robot. 2018; 00 (00).

[25] Callens SJP, Zadpoor AA. From flat sheets to curved geometries: Origami and kirigami approaches. Mater Today. 2018; 21(3): 241-264.

[26] Hwang D-G, Bartlett MD. Tunable Mechanical Metamaterials through Hybrid Kirigami Structures. Sci Rep. 2018; 8.

[27] Choi GPT, Dudte LH, Mahadevan L. Programming shape using kirigami tessellations. Nat Mater. 2019; 18: 9991004.

[28] Lee Y-J. Engineering the Deformation Characteristics of Soft Materials through Geometric Design of Auxetics for Flexible Electronics Application [PhD Dissertation]. South Korea: Department of Materials Science and Engineering, The Graduate School, Seoul National University; 2017.

[29] Shan S, Kang SH, Zhao Z, Fang L, Bertoldi K. Design of planar isotropic negative Poisson's ratio structures. Extreme Mech Lett. 2015; 4: 96-102.

[30] Zhang W, Neville R, Zhang D, Scarpa F, Wang L, Lakes R. The two-dimensional elasticity of a chiral hinge lattice metamaterial. Int J Solids Struct. 2018; 141-142: 254-263.

[31] Xu L, Shyu TC, Kotov NA. Origami and Kirigami Nanocomposites. ACS Nano. 2017; 11: 7587-7599.

[32] Vazquez AN, Jabi W. A Collaborative Approach to Digital Fabrication: A Case Study for the Design and Production of Concrete 'Pop-up' Structures. International Journal of Architectural Computing. 2015; 13(2): 195-216.

[33] Evans KE, Alderson A. Auxetic Materials: Functional Materials and Structures from Lateral Thinking! Adv Mater. $2000 ; 12(9): 617-628$.

[34] Konaković M, Crane K, Deng B, Bouaziz S, Piker D, Pauly M. Beyond Developable: Computational Design and Fabrication with Auxetic Materials. ACM Trans Graph. 2016; 35(4).

[35] Mesa O, Stavric M, Mhatre S, Grinham J, Norman S, Sayegh A, Bechthold M. Non-Linear Matters: Auxetic Surfaces. In: Proceedings of the 37th Annual Conference of the Association for Computer Aided Design in Architecture (ACADIA). Cambridge, USA; 2017: 392- 403.

[36] Grima JN, Evans KE. Auxetic behavior from rotating squares. J Mater Sci Lett. 2000; 19(17): 1563-1565.

[37] Grima JN, Evans KE. Auxetic behavior from rotating triangles. J Mater Sci. 2006; 41(10): 3193-3196.

[38] Lim T-C. Auxetic Materials and Structures. School of Science and Technology, SIM University, Singapoore: Springer; 2015.

[39] Louth H, Reeves D, Bhooshan S, Schumacher P, Koren B. A prefabricated dining pavilion: using structural skeletons, developable offset meshes and kerf-cut bent sheet material. In: Menges A, Scheil B, Glynn R, Skavara M, eds. Fabricate 2017: Rethinking design and construction. London: UCL Press; 2017. 58-67.

[40] Jacobsen JO, Howell LL, Magleby SP. Components for the design of Lamina Emergent Mechanisms. In: Proceedings of the 2007 ASME International Mechanical Engineering Congress and Exposition. United States; 2007.

[41] Jacobsen JO, Winder BG, Howell LL, Magleby SP. Lamina Emergent Mechanisms and Their Basic Elements. J Mech Robot. 2010; 2.

[42] Nelson TG, Lang RJ, Pehrson NA, Magleby SP, Howell LL. Facilitating Deployable Mechanisms and Structures Via Developable Lamina Emergent Arrays. J Mech Robot. 2016; 8.

[43] Howell LL. Compliant mechanisms. New York: John Wiley \& Sons; 2001.

[44] Nelson TG. Art to Engineering: Curved Folding and Developable Surfaces in Mechanism and Deployable Structure Design [PhD Dissertation]. Brigham Young University: Department of Mechanical Engineering; 2018.

[45] Wilding SE. Expanding Lamina Emergent Mechanism (LEM) Capabilities: Spherical LEMs, LEM Joints, and LEM Applications [Master Thesis]. Brigham Young University: Department of Mechanical Engineering; 2011. 
[46] Greenberg H. The Application of Origami to the Design of Lamina Emergent Mechanisms (LEMs) with Extensions to Collapsible, Compliant and Flat-Folding Mechanisms [Mater thesis]. Brigham Young University: Department of Mechanical Engineering; 2012.

[47] Delimont IL. Compliant Joints Suitable for use as Surrogate Folds [Master thesis]. Brigham Young University: Department of Mechanical Engineering; 2014.

[48] Delimont IL, Magleby SP, Howell LL. Evaluating Compliant Hinge Geometries for Origami-Inspired Mechanisms. J Mech Robot. 2015; 7: 1-8.

[49] Capone M, Lanzara E. Parametric Kerf Bending: Manufacturing Double Curvature Surfaces for Wooden Furniture Design. In: Bianconi F and Filippucci M, eds. Digital Wood Design. Lecture Notes in Civil Engineering 24. Springer, Cham; 2019. 415-439.

[50] Mansoori M, Kalantar N, Creasy T, Rybkowski Z. Adaptive Wooden Architecture: Designing a Wood Composite with Shape-Memory Behavior. In: Bianconi F and Filippucci M, eds. Digital Wood Design. Lecture Notes in Civil Engineering 24. Springer, Cham; 2019. 703-717.

[51] Menges A. Integrative Design computation: Integrating material behaviour and robotic manufacturing processes in computational design for performative wood constructions. In: Taron J, Parlac V, Kolarevic B and Johnson J, eds. Proceedings of the 31st Annual Conference of the Association for Computer Aided Design in Architecture. Banff, Canada; 2011: 72-81.

[52] Mitov D, Tepavčević B, Stojaković V, Bajšanski I. Kerf Bending Strategy for Thick Planar Sheet Materials. Nexus Netw J. 2019; 21: 149-160.

[53] Zarrinmehr S, Akleman E, Ettehad M, Kalantar N, Borhani A. Kerfing with Generalized 2D Meander-Patterns: Conversion of Planar Rigid Panels into Locally-Flexible Panels with Stiffness Control. In: Proceedings of the 17th International Conference CAAD Futures 2017. Istanbul, Turkey; 2017: 276-293.

[54] Gursoy B, Ozkar M. Visualizing making: Shapes, materials, and actions. Des Stud. 2015; 41: 29-50.

[55] Matini MR, Haghnazar R. Design of a curved-line bending mechanism inspired by skin wrinkles for elastic-kinetic structures in architecture. International Journal of Space Structures. 2018; 33 (3-4): 124-13.

[56] Holterman A. Pattern Kerfing for Responsive Wooden Surfaces. A formal approach to produce flexible panels with acoustic performance [Master thesis]. Netherlands: Delft University of Technology; 2018.

[57] Gomes AC. Kerf bending: técnica de flexão de madeira aplicada ao mobiliário [Undergraduate thesis]. Florianópolis, Brazil: Federal University of Santa Catarina, 2017.

[58] Gutowski SM. The bright side of failure: developing a set of lamps from an unsuccessful chair [Master thesis]. Iowa city, Iowa, USA: Graduate College of The University of Iowa; 2017.

[59] Lanzara E, Capone M. Kerf-Bending Tests: Design for Manufacturing Doubly Ruled Surfaces. In: Marcos C L, ed. Graphic Imprints. Springer, Cham; 2019. 1117-1130.

[60] Jensen MB, Blindheim J, Steinert M. Prototyping shape-changing interfaces - an evaluation of living hinges' abilities to resemble organic, shape-changing interfaces. In: Maier A, Škec S, Kim H, Kokkolaras M, Oehmen J, Fadel G, Salustri F, Van der Loos M, eds. Proceedings of the 21st International Conference on Engineering Design (ICED 17), v. 4. Design Methods and Tools, Vancouver, Canada; 2017: 287-296.

[61] Wei M, Singh K. Bend-a-rule: a fabrication-based workflow for 3D planar contour acquisition. In: Spencer SN, ed. Proceedings of the 1st Annual ACM Symposium on Computational Fabrication. Cambridge, USA; 2017.

[62] Güzelci OZ, Alaçam S, Bacinoğlu SZ. Three-step experimentation on embedding curvature to rigid planar materials through cut patterns. Gestão e Tecnologia de Projetos. 2017; 12(3): 93-107.

[63] Celli P, Mcmahan C, Ramirez B, Bauhofer A, Naify C, Hofmann D, Audoly B, Daraio C. Shape-morphing architected sheets with non-periodic cut patterns. Soft Matter. 2018; 14: 9744 - 9749. 